\title{
Navegação de VANT por Reconhecimento de Imagem do Solo
}

\author{
Fábio Luiz Junior* Leandro Aparecido Simal Moreira ${ }^{* *}$ \\ Jauvane Cavalcante de Oliveira ${ }^{* * *}$ \\ Paulo Fernando Ferreira Rosa ${ }^{* * * *}$ \\ * Programa de Pós-Graduação em Sistemas e Computação, Instituto \\ Militar de Engenharia, RJ, (e-mail: fabioluiz@ime.eb.br) \\ ** Programa de Pós-Graduação em Engenharia de Defesa, Instituto \\ Militar de Engenharia, RJ, (e-mail: leandromoreirar5@gmail.com) \\ *** Programa de Pós-Graduação em Modelagem Computacional, \\ Laboratório Nacional de Computação Científica, RJ, (e-mail: \\ jauvane@gmail.com) \\ **** Programa de Pós-Graduação em Engenharia de Defesa, Instituto \\ Militar de Engenharia, RJ, (e-mail: rpaulo@ime.eb.br)
}

\begin{abstract}
This paper implements a system capable of navigating a UAV (Unmanned Aerial Vehicle) using ground image recognition. For this, an experimental aircraft was developed and a 2-step algorithm implemented. In the first step, an acquisition flight and composition of image database of the ground is realized through the execution of any trajectory of flight. In the second step, using the image database of the trajectory, the aircraft is able to perform the same route again in an automated way using computational vision.

Resumo: Este artigo implementa um sistema capaz de realizar a navegação de um VANT (Veículo Aéreo Não Tripulado) utilizando reconhecimento de imagens do solo. Para isso, uma aeronave experimental foi desenvolvida e então implementado um algoritmo de 2 passos. No primeiro passo, realiza-se um voo de aquisição e composição de um banco de imagens do solo através da execução de uma trajetória qualquer de voo. No segundo passo, utilizando-se do banco de dados de imagens da trajetória e seu devido processamento, a aeronave é capaz de realizar novamente o mesmo trajeto de maneira automatizada utilizando visão computacional.
\end{abstract}

Keywords: Visual Navigation; Drone; Image Recognition; OpenCV; UAV.

Palavras-chaves: Navegação Visual; Drone; Reconhecimento de imagem; OpenCV; VANT.

\section{INTRODUÇÃO}

Os Veículos Aéreos Não Tripulados (VANTs) são aeronaves que não necessitam de um piloto humano a bordo, podendo ser controlados por um computador embarcado ou por um operador à distância. Nos últimos anos, os VANTs vem ganhando cada vez mais importância no cenário mundial devido às suas inúmeras possibilidades de aplicação. Neste contexto, as pesquisas sobre VANTs têm buscado introduzir um nível cada vez maior de inteligência embarcada, possibilitando que a máquina tome algumas decisões automaticamente, como o controle do seu trajeto de voo e a resposta aos estímulos do ambiente. Uma das linhas de pesquisas que está sendo empregada na automatização de voos é a utilização da visão computacional, a qual está em grande ascensão recentemente. Este artigo aborda alguns conceitos e realiza uma implementação para a navegação de um VANT utilizando o reconhecimento de imagens do solo. Para realizar os experimentos, construiu-se um VANT com um computador e uma câmera embarcados. Inicialmente, é realizado um voo para adquirir imagens do solo e compor um banco de dados com essas imagens. Em seguida, são realizados voos automatizados utilizando as imagens colhidas no primeiro voo. No controle da trajetória, é realizado o processamento em tempo real das informações obtidas pela câmera embarcada e, então, enviados comandos ao piloto automático da aeronave por meio do protocolo MavLink (MAVLink, 2019). Como contribuição obtida por este trabalho, destaca-se a pesquisa e desenvolvimento de meios alternativo de localização e navegação de VANTs. Pois este sistema é independente de mecanismos tradicionais de georreferenciamento por satélites.

O artigo está organizado do seguinte modo: a Seção 2 cita alguns trabalhos relacionados com o emprego de visão computacional em VANTs; a Seção 3 aborda dois conceitos importantes sobre reconhecimento de imagens; a Seção 4 descreve sucintamente a aeronave construída para este experimento; a Seção 5 explica o algoritmo implementado para a navegação do VANT utilizando o reconhecimento de imagens do solo; e a Seção 6 apresenta as conclusões.

\section{TRABALHOS RELACIONADOS}

Ao longo deste trabalho, foi realizada uma ampla pesquisa bibliográfica sobre o assunto na qual são destacados alguns pontos a seguir. Em Lu et al. (2018), constatou- 
se que a navegação baseada em visão provou ser uma promissora fonte de navegação devido ao rápido desenvolvimento da visão computacional nos últimos anos. Além disso, os sensores visuais, quando comparados a outros sensores como GPS e ultrassônicos, podem prover uma grande quantidade de informações do ambiente. Destacouse também que o método de rastreamento de keypoints nas imagens tem se tornado um robusto meio de localização e mapeamento. Em Cho et al. (2013), ressaltou-se que é possível determinar o movimento da cena detectando os movimentos relativos dos keypoints em uma sequência de imagens, porém tais métodos possuem como desvantagem a baixa performance em ambientes com pouca textura. Em Shen et al. (2014), os autores destacaram que a fusão de dados de múltiplos sensores pode prover grande melhora na performance da navegação. Em Leutenegger et al. (2015), enfatizou-se que a navegação utilizando sensores inerciais (Inertial Measurement Unit - IMU) conjuntamente com a visão computacional tem ganhado muita atenção nos últimos anos. Em Krajník et al. (2012), os autores construíram um algoritmo para estimar e controlar o ângulo de heading da aeronave, além de calcular sua velocidade vertical, ambos utilizando visão computacional. Para isso, embarcaram uma câmera monocular em um micro VANT e utilizaram o método SURF (Speeded-Up Robust Features) (Bay et al., 2006) para o cálculo dos keypoints e seus respectivos descriptors. Em Engel et al. (2012), os autores desenvolveram um algoritmo para o controle da estabilização da aeronave em ambientes indoor, sem sinal do GPS. As imagens capturadas eram transmitidas via WiFi a um computador externo, o qual realiza todo o processamento da informação e, então, envia os comandos de controle para a aeronave.

\section{CONCEITOS IMPORTANTES}

Nesta seção, serão discutidos alguns conceitos sobre o reconhecimento de imagens utilizando a biblioteca OpenCV (OpenCV, 2019). Tais conceitos são importantes para um melhor entendimento deste artigo.

Como abordado na introdução, o objetivo deste trabalho é realizar a navegação do VANT utilizando reconhecimento de imagens em tempo real obtidas através de uma câmera embarcada. Para isso, utilizou-se a biblioteca OpenCV que é desenhada para processamento de imagens, desenvolvida em $\mathrm{C} / \mathrm{C}++$ e com suporte à multi-threads. Além disso, possui interface com outras linguagens como Python e Java e pode ser executada nos principais sistemas operacionais do mercado.

Esta biblioteca é relativamente grande, possuindo diversas classes e métodos para os mais variados empregos em imagens e vídeos digitais. No entanto, neste artigo, só serão abordados os conceitos chaves de keypoint e descriptor necessários para o entendimento do restante do trabalho. Seguindo Kaehler and Bradski (2017a), keypoint é, em um alto nível de abstração, uma pequena porção da imagem que, por alguma razão, é usualmente distinta das demais e que se acredita ser possível localizá-la em uma outra imagem diferente. Em outras palavras, keypoint é uma pequena região da imagem com informações distintas comparando-se a outras áreas da mesma imagem. O descriptor é uma construção matemática, usualmente

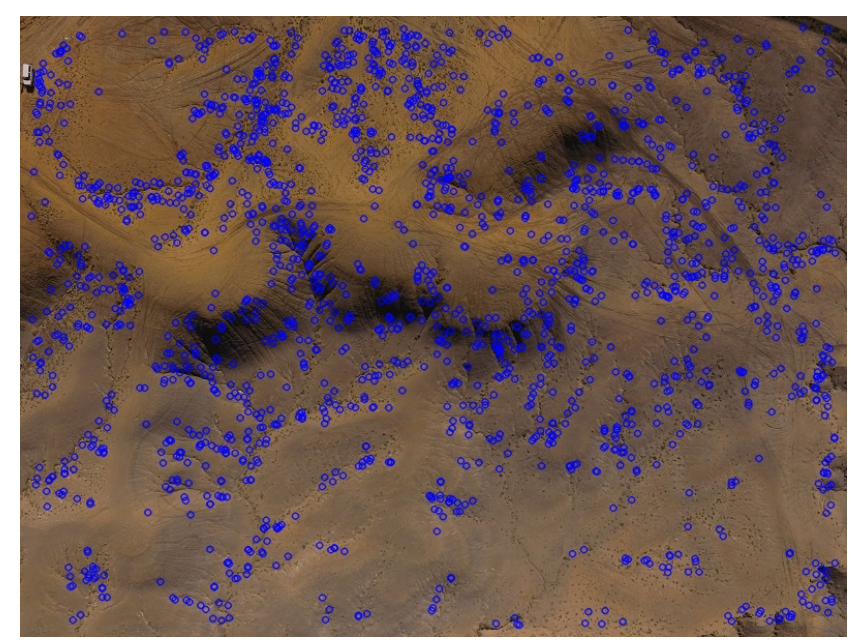

Figura 1. Destaque em azul dos keypoints de uma imagem aérea tirada por um VANT. Fonte: DroneMapper (2019).

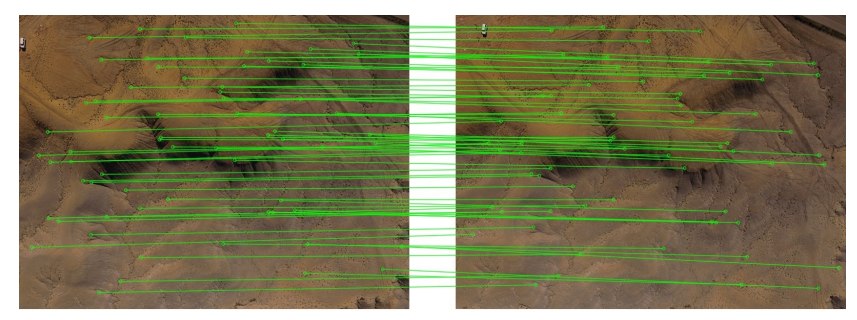

Figura 2. Associação de keypoints de duas imagens aéreas obtidas em diferentes voos de um VANT.

representada por um vetor de pontos flutuantes, que descreve e especifica um keypoint e que pode ser utilizado para determinar se dois keypoints extraídos de contextos diferentes são iguais ou parecidos. A utilização conjunta dos keypoints e seus descriptors permite criar uma impressão digital da imagem analisada, ou seja, processa a imagem criando uma sequência finita de números que a representa. Essa representação criada pode ser utilizada para comparar similaridades entre imagens ou a detecção de pedaços de uma em outra.

Como exemplo da utilização destes dois conceitos, na Figura 1 tem-se uma imagem aérea tirada por um VANT (DroneMapper, 2019). Nela, foram extraídos os seus keypoints e destacados em azul. Na Figura 2, utilizou-se os keypoints e seus descriptors para fazer uma análise de duas imagens obtidas de uma mesma área em diferentes voos de um VANT. Constata-se que, embora as imagens sejam diferentes, elas contém um alto grau de similaridade.

Por fim, o OpenCV possui vários métodos para manipular diversos tipos de keypoints e descriptors. Os algoritmos ou métodos mais conhecidos são: SIFT (Scale Invariant Feature Transform) (Lowe, 2004), SURF (Speeded-Up Robust Features) (Bay et al., 2006), ORB (Oriented FAST and Rotated BRIEF) (Rublee et al., 2011), FAST (Features from Accelerated Segments Test) (Rosten and Drummond, 2006), entre outros como descrito em Kaehler and Bradski (2017b). 


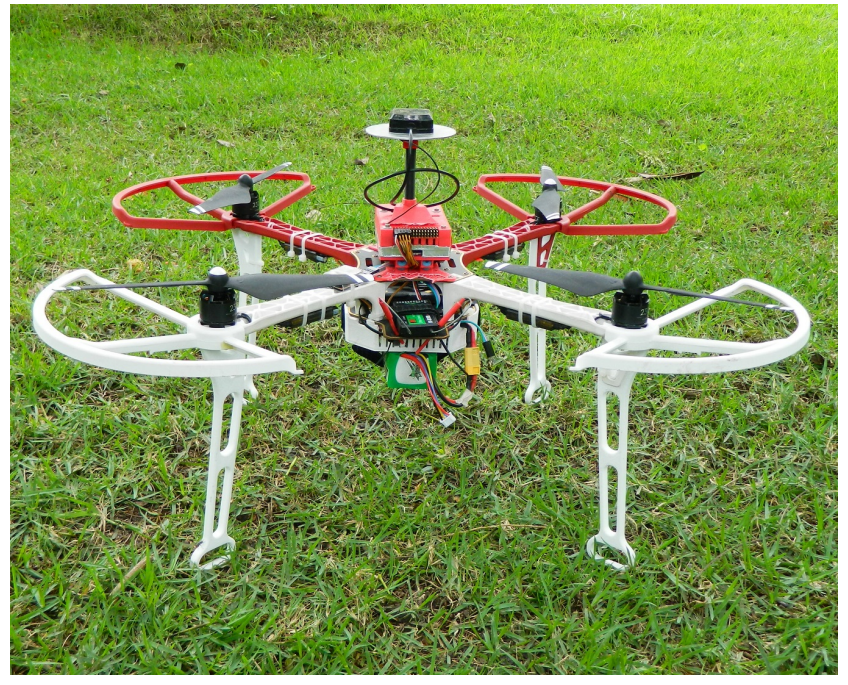

Figura 3. Vista lateral inclinada da aeronave desenvolvida para este trabalho.

\section{CONSTRUÇÃO DA AERONAVE}

A aeronave desenvolvida para este trabalho de pesquisa se encontra representada na Figura 3. O hardware utilizado é composto principalmente por uma placa Raspberry $\mathrm{Pi}$ 3 (RPI) (Foundation, 2019), uma placa Navio2 (Emlid, 2019), uma câmera integrada ao RPI de 5 megapixels, um gimbal de 2 eixos, 4 motores elétricos sem escova (brushless), 4 dispositivos de controle eletrônico de velocidade (Electronic Speed Control - ESC), um receptor de rádio controle, um rádio link para envio de comandos e recepção de telemetria (ArduPilot, 2019a), uma antena para o sistema de posicionamento por satélite e uma bateria. Como estrutura física do VANT, foi utilizado o frame $F 450$ da empresa DJI (DJI, 2019).

Sobre os componentes que compõe o sistema VANT é interessante destacar: (1) O RPI é o responsável pelo processamento embarcado da aeronave, possuindo 4 núcleos de processamento e executa uma versão customizada do Linux fornecido pelo fabricante da Navio2. (2) A placa Navio2 contém os sensores e mecanismos utilizados pelo piloto automático da aeronave. Tais sensores são: barômetro, acelerômetro, giroscópio, magnetômetro e sistema de posicionamento por satélite. (3) Embora presente no VANT, este trabalho não faz uso do GPS. (4) O Ardupilot (ArduPilot, 2019c) é o software de piloto automático da aeronave, sendo responsável pela leitura dos sensores, realização dos cálculos necessários para o controle físico do voo e atuação dos motores. (5) O Mission Planner (Oborne, 2019) é um software opcional para planejamento, em alto nível de abstração, do voo do VANT. Pode ser utilizado também para ler dados de telemetria, visualizar e atribuir configurações e status da aeronave, entre outras atribuições.

A arquitetura de funcionamento do sistema VANT está representada sucintamente na Figura 4. As setas azuis representam as comunicações entre os componentes. Em (i) e (ii), ocorrem a transmissão dos dados de telemetria, as configurações e o controle da aeronave. Em (iii), há a leitura dos dados dos sensores embarcados na aeronave. Em (iv), tem-se a saída do piloto automático para a

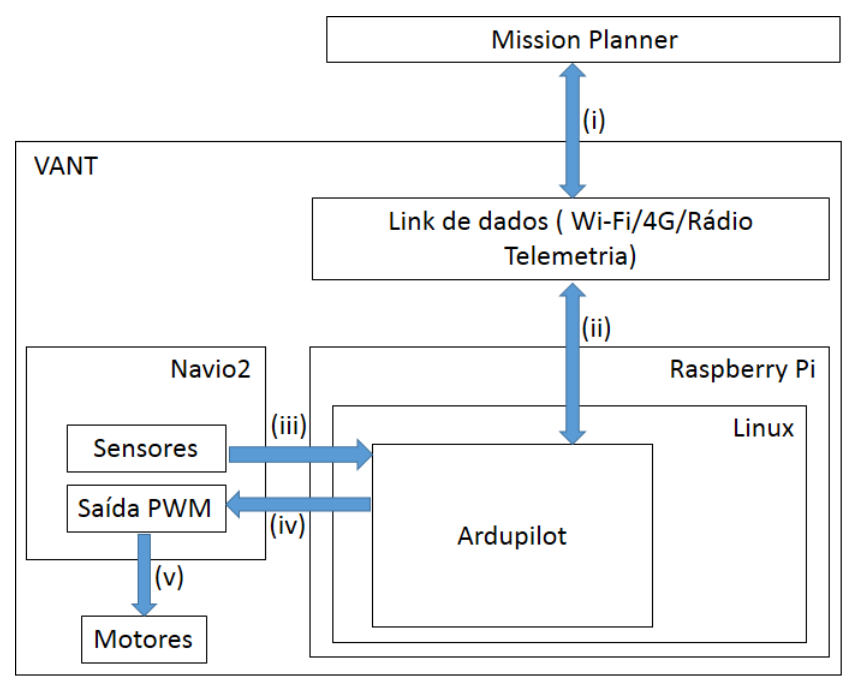

Figura 4. Arquitetura de funcionamento do sistema VANT. atuação dos motores. Em (v), tem-se os comandos elétricos aos motores.

\section{NAVEGAÇÃO POR RECONHECIMENTO DE IMAGEM DO SOLO}

Como já exposto, o objetivo deste trabalho é realizar voos automatizados sobre uma trajetória definida utilizando o reconhecimento de imagens do solo. Através da utilização dos mecanismos fornecidos pelo OpenCV, é possível criar um sistema de navegação do VANT utilizando imagens previamente carregadas no sistema. Tais imagens podem ser utilizadas como marcos visuais (waypoints) de uma trajetória que se deseja realizar. Assim, uma aeronave pode navegar seguindo uma sequência de marcos visuais de uma trajetória. Uma vez identificado um marco visual durante o voo, serão enviados comandos ao piloto automático para que a aeronave busque e centralize esse marco na imagem obtida em tempo real pela câmera embarcada. Uma vez alcançado o marco, o VANT irá buscar marcos de sequências superiores para o prosseguimento e execução do trajeto. A vantagem deste tipo de navegação, utilizando visão computacional, é a não necessidade de possuir um sistema de posicionamento por satélites, como o GPS, para a entrada de dados de localização no piloto automático. A desvantagem é a necessidade de haver textura no solo e que este não tenha grandes modificações entre os voos. A

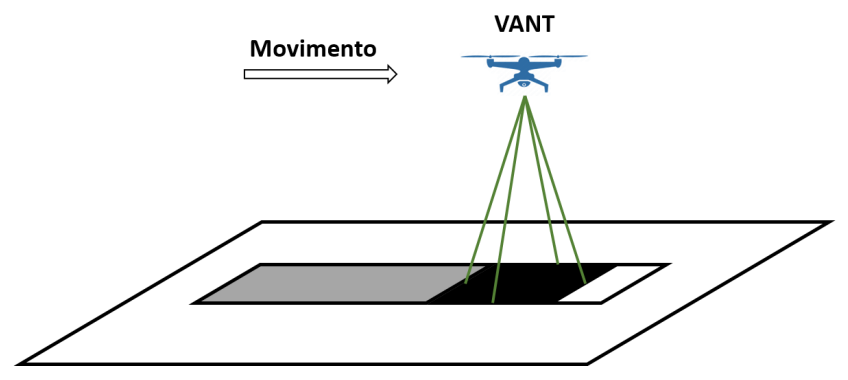

Terreno

Figura 5. Voo de captura das imagens do solo. Neste exemplo, é realizado um voo linear e as imagens são armazenadas em um banco de dados. 


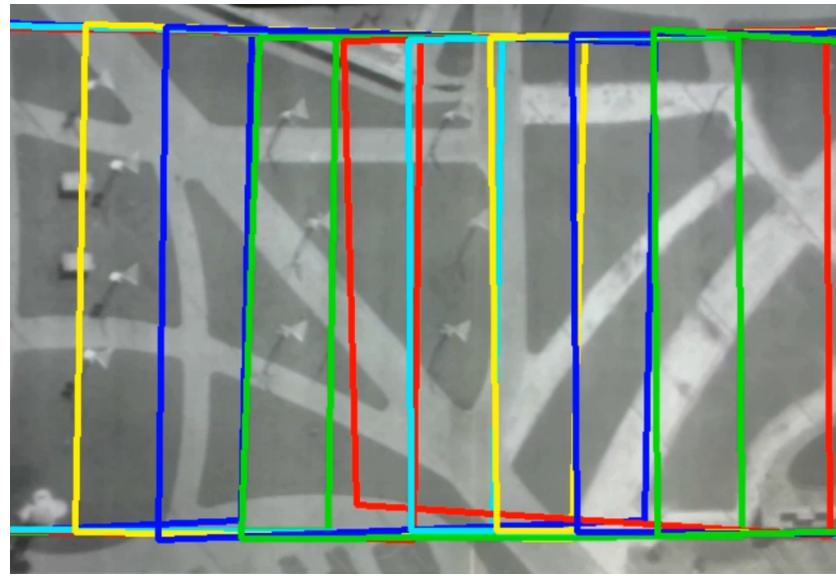

Figura 6. Identificação de diversos marcos visuais com um overlap de $83 \%$ entre eles.

Figura 5 ilustra de maneira simplificada um voo linear na etapa de captura dos marcos visuais. O retângulo cinza representa a parte do trajeto já capturada e processada pelo algoritmo e o quadrado preto representa a área de visibilidade atual pela câmera.

Para se alcançar o objetivo proposto por este trabalho, o sistema é executado em duas etapas. A primeira consiste de um voo manual para aquisição de imagens do terreno. Neste voo, um operador humano irá realizar um trajeto qualquer com a aeronave. Durante este trajeto, são obtidas diversas imagens do terreno abaixo utilizando a câmera embarcada disponível. Essa câmera fica sempre apontada para baixo, na vertical, através da utilização de um gimbal de dois eixos (paralelos ao plano do solo). Assim, o centro da imagem capturada pela câmera representa a projeção da posição do VANT sobre o terreno por onde se realiza a navegação. A taxa de captura das imagens deve ser ajustada de acordo com a velocidade de deslocamento do VANT, quanto maior for a sua velocidade, maior deverá ser a taxa de captura. Isso se deve pois, para o funcionamento do sistema implementado, é necessário que haja overlap entre as imagens capturadas. Este é necessário porque a visada da câmera deve capturar, na fase do voo autônomo, dois ou mais marcos visuais simultaneamente. As imagens são armazenadas em um banco de dados e podem ser processadas no momento do voo ou posteriormente para a extração dos keypoints e descriptors. Além disso, são numeradas em sequência de obtenção. Após o processamento, os arquivos originais das imagens podem ser descartados, já que os reconhecimentos posteriores serão realizados com base nas características extraídas da imagem (keypoints e descriptors). O método utilizado para o reconhecimento é o SURF, disponível na biblioteca OpenCV.

A segunda etapa consiste do voo automatizado utilizando as imagens processadas da primeira etapa. Os voos automatizados, desta segunda etapa, poderão ser realizados inúmeras vezes até que ocorra a modificação física do terreno sobre o qual se realizou o voo de aquisição das imagens. Para a execução do voo autônomo, inicialmente os dados dos marcos visuais (keypoints e descriptors) devem ser carregados na aeronave e esta posta em algum ponto da trajetória que se deseja refazer. Após isso, a aeronave decola perpendicularmente ao ponto até encontrar um dos

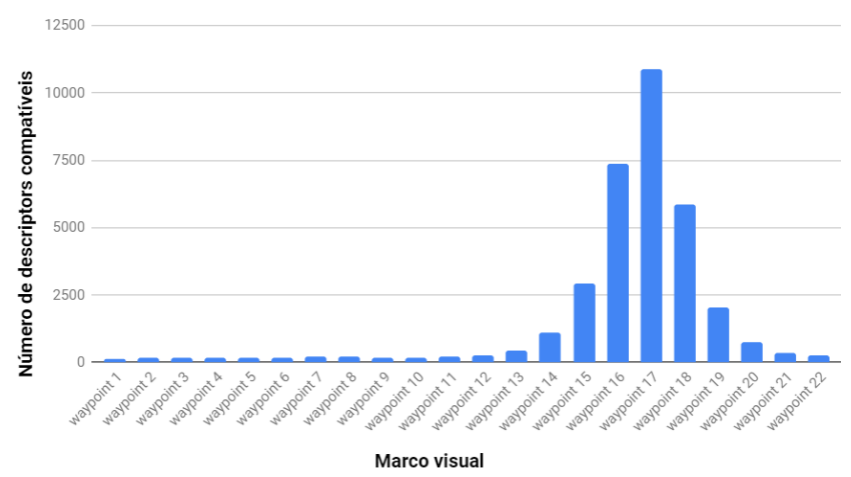

Figura 7. Comparação dos keypoints de uma imagem adquirida pela câmera embarcada com os dos marcos visuais constantes no banco de dados da trajetória.

marcos visuais na imagem obtida da câmera embarcada. Visualizando um marco, a aeronave irá executar comandos para alcançar esse marco, posicionando o centro da imagem capturada pela câmera no centro do marco visual já salvo. Nota-se que os marcos são armazenados no banco de dados conjuntamente com seus números de sequência. Assim, na estratégia de perseguição destes, a aeronave irá privilegiar aqueles com maiores números de sequência. Ou seja, caso a câmera capture simultaneamente os marcos visuais 40 e 41, o sistema irá perseguir o marco 41 em detrimento ao marco 40. Isso fará com que a trajetória do primeiro voo seja refeita na ordem original. Como já dito, há a necessidade de overlap entre os marcos visuais, para que o sistema identifique alguns marcos em uma mesma imagem capturada. A Figura 6 contém um exemplo da identificação de diversos marcos visuais com um overlap de $83 \%$ entre eles.

A seguir, para melhor entendimento e descrição do mecanismo utilizado, este capítulo foi dividido em algumas Subseções. Em cada uma, serão explicados alguns aspectos sobre o funcionamento do sistema.

\subsection{Processamento das imagens e busca dos marcos visuais no banco de dados}

Como anteriormente citado, os keypoints e descriptors são extraídos da imagem utilizando o algoritmo SURF do OpenCV. Este método foi escolhido pois apresenta um bom desempenho computacional, além de ser invariante à mudança de perspectiva das imagens analisadas. As informações resultantes do processamento das imagens obtidas são, então, salvas em um banco de dados que compõe a trajetória que se deseja refazer nos voos subsequentes. Com relação à busca pelos marcos visuais, esta ocorre através da comparação entre os keypoints das imagens adquiridas em tempo real pela câmera embarcada e os dados constantes em tal banco de marcos visuais. Para isso, compara-se, utilizando os descriptors, a quantidade de keypoints semelhantes entre as imagens. Quanto maior o número de keypoints semelhantes ou próximos, maior será a semelhança das imagens analisadas. Configura-se, então, um limiar de aceitação com base na quantidade de keypoints semelhantes, a partir do qual se pode assumir que as imagens são as mesmas. 
O gráfico da Figura 7 representa a quantidade de keypoints semelhantes entre uma imagem capturada pela câmera e os marcos visuais do banco de dados. No eixo vertical, tem-se expresso o número de descriptors semelhantes ou compatíveis. No eixo horizontal, tem-se os marcos visuais armazenados no banco de dados. Da análise do pico central do gráfico, verifica-se que o marco visual mais próximo à imagem obtida pela câmera é o waypoint 17. Muito embora os waypoints 16 e 18 também possuam um alto grau de semelhança com a imagem analisada. Isso se deve pois, como há overlap entre os marcos visuais, eles compartilham uma certa quantidade de keypoints.

\subsection{Cálculo do movimento da aeronave}

Neste trabalho, a fim de diminuir a complexidade do sistema de cálculo da trajetória, restringiu-se o movimento da aeronave a um plano paralelo ao solo, mantendo-se a altura de voo constante. Para isso, utilizou-se o modo de voo AltHold disponível no Ardupilot (ArduPilot, 2019b). Neste modo, é possível controlar a atitude da aeronave (roll, pitch e yaw) normalmente, enquanto o piloto automático mantém a altitude constante através da leitura dos dados do barômetro. Assim, com movimentos paralelos ao solo, os comandos enviados para a aeronave podem ser decompostos em dois tipos, um comando de rotação e um de translação. Executados nesta ordem e sucessivamente para cada marco visual identificado. Ou seja, logo que ocorra a identificação de um marco visual, a aeronave irá rotacionar até alcançar o alinhamento com este marco, alinhamento esse entre as imagens capturada em tempo real pela câmera e a imagem processada do bando de dados. E, após alinhada, irá fazer um movimento de translação para posicionar o centro da imagem obtida pela câmera sobre o centro da imagem representada pelo marco visual. Na Figura 8, destaca-se o reconhecimento do waypoint 19. Este é demarcado pelo quadrado com a borda em vermelho. A seta vermelha representa o vetor deslocamento instantâneo para se alcançar o marco visual 19. Esta possui como origem o centro da imagem, que representa a posição atual do VANT, e aponta para o centro do marco visual identificado. A seta verde resulta do tratamento estatístico dado aos vetores instantâneos em vermelho, o qual será visto na subseção 5.3. O número 0,3 representado no centro da imagem, indica a quantidade em graus que é necessário rotacionar a aeronave para realizar o alinhamento desta com o marco visual 19 (waypoint). Lembrando-se que os marcos são numerados em sequência crescente. Assim, tão logo o seguinte marco visual esteja visível pela câmera e identificado pelo algoritmo, a aeronave irá realizar a sequência de rotação e translação para alcançá-lo. Fazendo-se com isso, que a aeronave percorra todo o trajeto programado pelos marcos visuais.

Abaixo são descritos os métodos utilizados para o cálculo da rotação e do vetor deslocamento necessários para o movimento do VANT.

Rotação da aeronave: O ângulo de rotação necessário a ser aplicado à aeronave é calculado através da imagem obtida em tempo real da câmera embarcada e da imagem processada anteriormente do marco visual. Para isso, utiliza-se a função findHomography(), disponível no OpenCV, que calcula a matriz homográfica. Esta matriz contém uma relação do mapeamento entre as duas imagens, a da câmera

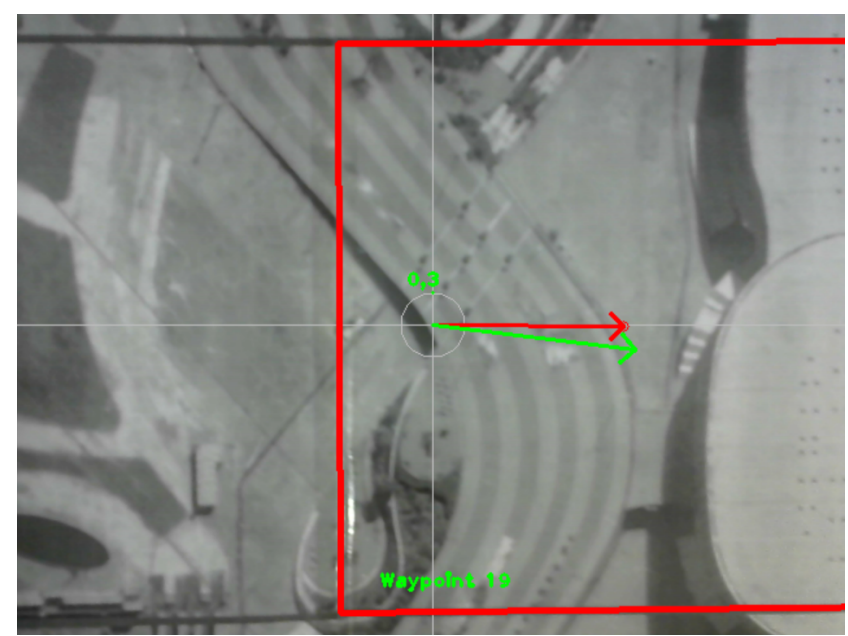

Figura 8. Identificação do marco visual 19, com a indicação do ângulo de rotação e do vetor deslocamento para o seu alinhamento com a aeronave.

e a do marco visual. Através dela pode-se obter várias informações interessantes. Uma delas é o ângulo relativo de rotação entre as duas imagens. A matriz homográfica possui dimensão $3 \times 3$ e está genericamente representada pela Equação 1.

$$
M_{3,3}=\left(\begin{array}{ccc}
m_{1,1} & m_{1,2} & m_{1,3} \\
m_{2,1} & m_{2,2} & m_{2,3} \\
m_{3,1} & m_{m, 2} & m_{3,3}
\end{array}\right)
$$

O ângulo de rotação relativo entre as duas imagens pode ser encontrado utilizando a Equação 2, onde $\alpha$ representa o ângulo em graus, $m_{1,1}$ e $m_{1,2}$ são elementos da matriz homográfica acima representada.

$$
\alpha=\left(\frac{180}{\pi}\right) \arctan \left(\frac{m_{1,2}}{m_{1,1}}\right)
$$

Na Figura 8, o ângulo de rotação está representado no centro da imagem e a sua magnitude é de 0,3 graus.

Vetor deslocamento: O vetor deslocamento da aeronave é calculado utilizando-se o centro da imagem obtida em tempo real, que representa a posição do VANT, e o centro do marco visual identificado. Na Figura 8, o quadrado vermelho indica que foi encontrado e identificado o waypoint 19. A seta vermelha representa o vetor deslocamento apontando do centro da imagem da câmera para o centro do waypoint identificado. Durante a execução do trajeto, caso um waypoint seguinte seja identificado (de índice superior), a aeronave irá passar a ser atraída para esse waypoint e não mais para o anterior.

\subsection{Filtragem das leituras realizadas}

Durante a execução do algoritmo aqui implementado, ocorreram erros no reconhecimento dos marcos visuais. Para minimizar esses erros e suavizar os movimentos da aeronave, utilizaram-se duas estratégias descritas a seguir. A primeira é a utilização de uma média móvel de 10 amostras, aplicadas tanto nas leituras do ângulo de rotação quanto nas do vetor deslocamento. Assim, leituras erradas dentro de um conjunto de várias leituras corretas não 
possuem uma forte influência no movimento da aeronave. Em contra partida, a aeronave demora mais tempo para responder aos marcos visuais reconhecidos. A segunda estratégia, utilizada conjuntamente com a primeira, é descartar rotações com valores superiores a $50 \%$ em relação à média. Já no caso do vetor deslocamento, descartam-se vetores com ângulo maiores a 90 também em relação à média. Ou seja, com isso faz-se uma filtragem de valores espúrios. Tais valores obtidos fora desse intervalo são possivelmente marcos visuais reconhecidos erradamente pelo algoritmo. A natureza dessa filtragem decorre das próprias restrições físicas da aeronave. Já que dificilmente haverá grandes alterações no seu movimento, deslocamento e ângulo de rotação, dentro de pequenos intervalos de tempo. Outra abordagem mais sofisticada é a utilização de Filtro de Kalman Estendido, como em Engel et al. (2012) e em Krajník et al. (2012).

\subsection{Execução dos comandos de movimento}

Após o cálculo da rotação e do vetor deslocamento necessários ao movimento da aeronave, faz-se em seguida a emissão dos comandos ao piloto automático para a sua execução. Para isso, pode-se utilizar uma abordagem em baixo nível, através do envio de comandos diretos pelo protocolo MavLink (MAVLink, 2019). A vantagem desse método é que se possui maior controle sobre a aeronave. Porém, necessita-se de um maior esforço de programação. A segunda abordagem é utilizar uma biblioteca de alto nível como a DroneKit (Robotics, 2019), disponível na linguagem Python e Java para Android. Nesse caso, perdese um pouco a liberdade de programação, mas gasta-se menos esforço de programação. Para os fins deste artigo, ambas são possíveis de utilização para controlar a atitude da aeronave e a leitura dos seus parâmetros de voo.

\subsection{Desempenho}

Nesta subseção, serão apresentadas as otimizações implementadas para melhorar o desempenho do sistema de navegação por imagem. Dependendo do tamanho do trajeto a ser realizado pela aeronave, poderá ser necessário armazenar em memória um grande conjunto de keypoints e descriptors dos marcos visuais processados. A busca de uma imagem obtida pela câmera dentro desse conjunto de marcos visuais pode resultar em grande necessidade de processamento. Um meio de otimizar esse processamento de procura e reconhecimento dos marcos, é restringir a janela de busca para uma vizinhança do último marco visual identificado. Por exemplo, se a última imagem do trajeto reconhecida foi a de número 500, busca-se a próxima imagem da câmera no intervalo de 498 a 502 . Isso permite a aeronave avançar ou retroceder no trajeto pretendido. Em outras palavras, caso a aeronave se encontre no marco visual de número 502, o centro da janela de busca da próxima imagem ocorre no intervalo de 500 a 504. Caso o marco visual 503 seja encontrado pela aeronave, a janela de busca será movida para o intervalo de 501 a 505.

Outra otimização realizada é na escolha da resolução das imagens utilizadas. Através de diversos testes realizados, optou-se pela resolução 640x480. Esta apresenta uma boa taxa de quadros por segundo de capacidade de processamento e uma boa taxa de reconhecimento dos marcos visuais analisados. Na Tabela 1, há uma comparação dos métodos utilizados, as resoluções das imagens e as taxas de quadros por segundo (frames per second - fps).

Tabela 1. Tabela comparativa do desempenho dos algoritmos SURF e SIFT para diferentes resoluções

\begin{tabular}{ccc} 
Método & Resolução & Taxa \\
\hline SURF & $320 \times 240$ & $14,6 \mathrm{fps}$ \\
SURF & $640 \times 480$ & $5,9 \mathrm{fps}$ \\
SURF & $1280 \times 720$ & $2,6 \mathrm{fps}$ \\
\hline SIFT & $320 \times 240$ & $12,7 \mathrm{fps}$ \\
SIFT & $640 \times 480$ & $4,4 \mathrm{fps}$ \\
SIFT & $1280 \times 720$ & $1,7 \mathrm{fps}$ \\
\hline
\end{tabular}

\section{CONCLUSÃO}

Através da pesquisa bibliográfica realizada durante este trabalho, verificou-se a viabilidade da construção de uma aeronave guiada por visão computacional. Com este objetivo, foi então montada e configurada uma aeronave para testes. Consecutivamente, implementou-se um algoritmo para a navegação do VANT utilizando o reconhecimento de marcos visuais do solo. O sistema criado realiza a aquisição dos marcos visuais através de um voo preliminar de captura das imagens. E, posteriormente, o sistema realiza voos automatizados através do reconhecimento e perseguição de sequências de marcos visuais utilizando a câmera embarcada da aeronave. O método escolhido para a extração das características das imagens e a busca pelos marcos foi o SURF disponível no OpenCV. Alguns testes realizados em laboratório indicam que a resolução de 640x480 possui uma boa relação entre desempenho na execução e reconhecimento dos marcos visualizados pela câmera. Implementaram-se também alguns métodos para a otimização na busca dos marcos visuais e a eliminação de dados espúrios. Por fim, realizou-se a integração dos comandos gerados pelo algoritmo, sequências de movimentos de rotação e de translação, com o piloto automático da aeronave. Para tal integração, utilizou-se o protocolo MavLink, compatível com o piloto automático Ardupilot utilizado na aeronave.

\section{AGRADECIMENTOS}

O presente trabalho foi realizado com apoio da Coordenação de Aperfeiçoamento de Pessoal de Nível Superior Brasil (CAPES) - Código de Financiamento 001.

\section{REFERÊNCIAS}

ArduPilot (2019a). 3dr radio. URL http://ardupilot. org/copter/docs/common-3dr-radio-v1.html.

ArduPilot (2019b). Altitude hold mode - copter documentation. URL http://ardupilot.org/copter/ docs/altholdmode.html\#altholdmode.

ArduPilot (2019c). Community: Ardupilot documentation. URL http://ardupilot.org/ardupilot/.

Bay, H., Tuytelaars, T., and Van Gool, L. (2006). Surf: Speeded up robust features. In European conference on computer vision, 404-417. Springer.

Cho, D.M., Tsiotras, P., Zhang, G., and Holzinger, M. (2013). Robust feature detection, acquisition and tracking for relative navigation in space with a known target. 
In AIAA Guidance, Navigation, and Control (GNC) Conference, 5197.

DJI (2019). Dji - the world leader in camera drones/quadcopters for aerial photography. URL https:// www.dji.com/flame-wheel-arf/spec.

DroneMapper (2019). Drone mapping aerial imagery example data - dronemapper. URL https:// dronemapper.com/sample_data/.

Emlid (2019). Emlid navio2 — raspberry pi autopilot hat powered by ardupilot. URL https://emlid.com.br/ navio/.

Engel, J., Sturm, J., and Cremers, D. (2012). Camerabased navigation of a low-cost quadrocopter. In Intelligent Robots and Systems (IROS), 2012 IEEE/RSJ International Conference on, 2815-2821. IEEE.

Foundation, T.R.P. (2019). Raspberry pi - teach, learn, and make with raspberry pi. URL https://www . raspberrypi.org/.

Kaehler, A. and Bradski, G. (2017a). Learning OpenCV 3, Computer Vision in $\mathrm{C}++$ with the OpenCV Library. O'Reilly Media Inc., United States of America, 1 edition.

Kaehler, A. and Bradski, G. (2017b). Learning OpenCV 3, Computer Vision in $\mathrm{C}++$ with the OpenCV Library. O'Reilly Media Inc., United States of America, 1 edition.

Krajník, T., Nitsche, M., Pedre, S., Přeučil, L., and Mejail, M.E. (2012). A simple visual navigation system for an uav. In Systems, Signals and Devices (SSD), 2012 9th International Multi-Conference on, 1-6. IEEE.

Leutenegger, S., Lynen, S., Bosse, M., Siegwart, R., and Furgale, P. (2015). Keyframe-based visual-inertial odometry using nonlinear optimization. The International Journal of Robotics Research, 34(3), 314-334.

Lowe, D.G. (2004). Distinctive image features from scaleinvariant keypoints. International journal of computer vision, 60(2), 91-110.

Lu, Y., Xue, Z., Xia, G.S., and Zhang, L. (2018). A survey on vision-based uav navigation. Geo-spatial information science, 21(1), 21-32.

MAVLink (2019). Mavlink developer guide. URL https : //mavlink.io/en/.

Oborne, M. (2019). Mission planner home - mission planner documentation. URL http://ardupilot.org/ planner/index.html.

OpenCV (2019). Opencv library. URL https://opencv . org/.

Robotics, D. (2019). Welcome to dronekit-python's documentation! URL http://python.dronekit.io/.

Rosten, E. and Drummond, T. (2006). Machine learning for high-speed corner detection. In European conference on computer vision, 430-443. Springer.

Rublee, E., Rabaud, V., Konolige, K., and Bradski, G.R. (2011). Orb: An efficient alternative to sift or surf. In $I C C V$, volume 11, 2. Citeseer.

Shen, S., Mulgaonkar, Y., Michael, N., and Kumar, V. (2014). Multi-sensor fusion for robust autonomous flight in indoor and outdoor environments with a rotorcraft mav. In Robotics and Automation (ICRA), 2014 IEEE International Conference on, 4974-4981. IEEE. 